\title{
Concentración de cobre en el suero de recién nacidos.
}

\author{
Dr. Sergio Krause H. ${ }^{\mathbf{1}}$, Sr. Antonio Rivas B. ${ }^{2}$ Dr. Fernando Olavar ría U. ${ }^{1}$ \\ Sra. Gester Perdiguero $\mathrm{N}^{3}$ y Srta. Kora Leal A.
}

\section{Serum Copper Levels in newbron infants}

\begin{abstract}
Serum copper levels were determined in 99 Newborn infants and their mothers Mean values oblained in newborns of gestational age 38 weeks or plus, were $53.7 \pm 5.8 \mathrm{~kg} / \mathrm{dl}$. $(X \pm S . D$.), those of 34 to 37 wecks: $41.7 \pm 4.1 \mathrm{\mu g} / \mathrm{dl}$, and for 27 to 33 week $33.8 \pm 1.7 \mathrm{~kg} / \mathrm{dl}$. Significant differences were observed between preterm and term infants.

There was a direct correlation $(r=0.707)$ between serum copper levels and gestational age, with influence of height and weight of neonates. No significant differences were observed for sexes or for different weights at the same gestational age. There was nat correlation between maternal serum copper levels and those obtained in neonates. Preterm infants should have lower serum copper levels, and be at more risk of copper deficiences.
\end{abstract}

El cobre es un elemento traza que se ha definido como esencial para el ser humano. Cumple funciones de biocatalizador metabólico y forma parte de algunas metaloenzimas, tales como citocromo c oxidasa, superoxidismutasa, tirosinasa, ceruloplasmina y lisiloxidasa, entre otras ${ }^{1-4}$.

1 Instituto de Pediauría, Fac. Medicina, Universidad Austral de Chile.

2 Bioquimico, Fac. Ciencias Agrarias, Universidad Austral de Chile.

3 Matrona, Hospital Base de Valdivia.

4 Enfermera, Hospital Base de Valdivia.

Presentado como trabajo de incorporación a la Sociedad Chilena de Rediatría del Dr. Sergio Krause H.
Еn 1964 se describió por primera vez el déficit nutricional de cobre ${ }^{1}$ y posteriormente se ha comunicado en pacientes con malabsorción intestinal $^{5}$, lactantes con antecedentes de premature $z^{6-7}$. en nutrición parenteral total sin suplemento de cobre $^{\mathrm{\theta} \cdot 9}$, en dietas pobres en cobre ${ }^{10} \mathrm{y}$ en lactantes desnutridos ${ }^{11}$. La deficiencta de cobre en el organismo es capaz de originar ciertas manifestaciones clínicas que están relacionadas con las alteraciones de las cuproenzimas antes mencionadas. Las manifestaciones más frecuentes de falta de cobre son: neutropenia, leucopenia, anemia, alteraciones óseas que pueden llevar a fracturas patológicas y trastornos neurológicos y vasculares ${ }^{6}-7-9$ $10 \cdot 12$. 
Como se han descrito manifestaciones clinicas por deficiencia de cobre en lactantes menores con antecedentes de premature $z$, se ha sugerido que la edad gestacional al nacer puede ser un factor innportante en la formación de reservas de este elemento. Asi los prematuros podrían desarrollar un balance negativo de cobre en los primeros meses de vida ${ }^{3-6-13}$. Sin embargo, el estado nutricional de cobre en los recién nacidos, su interacción con factores dietarios, y su proyección y significado no están claramente deteminados ${ }^{3-13 \cdot 14}$. En Chile desconocemos información referente al estado nutricional de cobre en recièn nacidos.

Este estudio pretende describir las concentraciones de cobre en el suero (cupremia) en recién nacidos, en relación con la edad gestacional y otras variables, así como los niveles de cupremia materпа.

\begin{tabular}{|c|} 
ABREVIATURAS USADAS \\
RNT $\quad \begin{array}{c}\text { : recién nacido de término }(38 \text { a } 42 \text { sema- } \\
\text { nas) })^{15}\end{array}$ \\
RNpreT : recién nacido de pretémino (37 o menos \\
semanas) \\
RNposT : rccién nacido de post-término (43 o \\
más semanas) \\
AEG : adecuado para la edad gestacional \\
PEG : pequeño para la edad gestacional \\
GEG : grande para la edad gestacional.
\end{tabular}

\section{MATERIAL Y METODOS}

Se estudiaron 99 recién nacidos (48 varones y 51 mujeres) y sus respectivas madres, del Hospital Base de Valdivia entre los meses de Marzo y Julio de 1982. Previamente se obtuvo consentimiento de los padres en cada caso. Las edades gestacionales fueron determinadas, según el método de Dubowitz, por el mismo médico para todos los recién nacidos.

Entre 12 y 24 horas después del parto, se tomó muestra de sangre venosa por punción, tanto al recién nacido como a su madre. Al tomar la muestra se completó un formulario que incluia datos de la madre (edad, paridad, peso y estatura) y del recién nacido (sexo, edad gestacional, peso y talla).

Las muestras de sangre fueron centrifugadas $y$, el suero correspondiente se diluyó con agua desionizada, se almacenó en tubos de plástico libres de cobre y se congeló para su posterior análisis. Las muestras hemolizadas fueron rechazadas. La determinación de los niveles de cobre sérico se realizó en un espectrofotómetro de absorción atómica Perkin-Elmer, Modeło 403, usando llama de aire-acetileno ${ }^{16}$.

El análisis estadístico comprendió promedio y desviación estandar ( $\bar{x} \pm \mathrm{D} . S$. ), coeficiente de corre- lación de Parson con la curva de regresión correspondiente y análisis de regresión múltiple. Los significados se determinaron por pruebas de Student y de Scheffe, fijándose la probabilidad de error en menos de $1 \% 17$.

\section{RESULTADOS}

Las características generales del grupo estudiado, se muestran en la Tabla 1 . El nivel promedio de cobre sérico en los 99 recién nacidos fue $50.1 \pm$ $8.1 \mu \mathrm{g} / \mathrm{dl}$. con un rango de 32 a $67 \mu \mathrm{g} / \mathrm{dl}$. En las madres correspondió a $234.3 \pm 39.1 \mu \mathrm{g} / \mathrm{dl}$. con rango de 139 a $340 \mu \mathrm{g} / \mathrm{dl}$.

Tabla 1.

Promedio, D.S. $y$ rangos de las características generales maternas y del recién nacido.

\begin{tabular}{|c|c|c|c|c|}
\hline & \multicolumn{2}{|c|}{$x=0 \mathrm{~S}$} & \multicolumn{2}{|c|}{ RANGO } \\
\hline \multicolumn{5}{|l|}{ MATERNAS } \\
\hline Edad (años) & $23.7:$ & 5.5 & 15 & a 43 \\
\hline Paridad & $1.3 \pm$ & 1.4 & 0 & a 7 \\
\hline Peso (kgs) & $58.3 \pm$ & 9.7 & 40.1 & $\square$ פดล \\
\hline Talla (cms) & $151.9 \pm$ & 6.4 & 134 & a 166 \\
\hline Relación PIT $\left(\psi^{*}\right)^{*}$ & $113.0 \pm$ & 16.2 & g) & a 184 \\
\hline Cobre sérico $(\mu g / d l)$ & $234.3 \pm$ & 39.1 & 139 & $\triangle 340$ \\
\hline \multicolumn{5}{|l|}{ RECIEN NACIDO } \\
\hline Edad gestacional (seman) & $38.5 \pm$ & 2.5 & 27 & a 43 \\
\hline Peso nacimiento (kgs) & $3.07 \pm$ & 0.70 & 0.81 & a 4.50 \\
\hline Talla nacimienlo (ems) & $48.3 \div$ & 3.1 & 33.0 & a 54.5 \\
\hline Cobre Serieo( $\mu \mathrm{g} / \mathrm{dl})$ & $50.1 \pm$ & 8.1 & 32 & 067 \\
\hline
\end{tabular}

En los recién nacidos no se encontraron diferencias significativas en los niveles de cobre sérico según sexo, siendo de $48.2 \pm 8.5 \mu \mathrm{g} / \mathrm{dl}$. para los varones y de $51.9 \pm 7.2 \mu \mathrm{g} / \mathrm{dl}$. en las mujeres.

Tampoco se observaron diferencias significativas en las variables peso, talla y edad gestacional, segün el sexo de los recién nacidos.

Al establecer categorías de edad gestacional en los recién nacidos y aplicar una prueba de Scheffe (Tabla 2), se observaron diferencias significativas $(p<0.01)$ entre las tres categorías para las variables peso y talla. El nivel promedio de cobre sérico en la categoría 38 o más. semanas $(53.7 \pm 5.8$ $\mu \mathrm{g} / \mathrm{dl}$.) fue significativamente diferente $(\mathrm{p}<0.01)$ de las cupremias de las otras dos categorías, las que no mostraron diferencias entre sí.

En el grupo de los recién nacidos de término, al distribuir los pesos y edades gestacionales según la clasificación de Battaglia y Lubchenco ${ }^{15}$ se demostró que no existian diferencias significativas en los valores de cobre sérico, según sean RNTAEG, RNTPEG o RNTGEG (Tabla 3). En este estudio 
sólo hubo un recién nacido de post-término (AEG) con 43 semanas de edad gestacional, su cupremia era de $64 \mu \mathrm{g} / \mathrm{d}$ l. y no fue incluido en la tabla antes mencionada.

Tabla 2.

Peso, talla y cobre sérico en recién nacidos según categorias de edad gestacional. Valores cxpresados en X \pm D S.

\begin{tabular}{|c|c|c|c|c|}
\hline $\begin{array}{l}\text { EDAD } \\
\text { GESTACHONAL }\end{array}$ & $\begin{array}{l}\text { NNo DE } \\
\text { CASOS }\end{array}$ & $\begin{array}{c}\text { PESO NAC. } \\
\text { (KgS) }\end{array}$ & $\begin{array}{c}\text { TALLA NAC. } \\
\text { ( Ims) }\end{array}$ & $\begin{array}{l}\text { COBRE SERICO } \\
(N g / d 1)\end{array}$ \\
\hline $\begin{array}{c}27 \text { a } 33 \text { semanas } \\
(\bar{x}=31.3)\end{array}$ & 4 & $1.61 \div 0.57$ & $40.4 \pm 4.9^{x}$ & $33.8 \pm 13$ \\
\hline $\begin{array}{c}34 \text { a } 37 \text { sermanas } \\
(\bar{x}=36.0)\end{array}$ & 23 & $2.50 \div 0.45$ & $45.6 \div 2.2^{x}$ & $41.7 \pm 4.1$ \\
\hline $\begin{array}{c}39 \text { orás semanas } \\
(\dot{X}=39.8)\end{array}$ & 72 & $3.34 \div 0.53^{\circ}$ & $\left\langle 9.7 \pm 1.8^{x}\right.$ & $53.7 \div 58{ }^{*}$ \\
\hline
\end{tabular}

- Diterencias significativas $1 p<0.01$ )

$x$ Dilerencios significativos $(p<0$.01)

* Difiere significativameniz $(p<0.01)$ de las olras 2 calegorias

\section{Tabla 3.}

Edad gestacional, peso, talla y cobte sérico en 71 R.N.T. según clasificación peso vs cdad gescacional. Valores expresad ox en $\bar{X} \pm$ D.S.

\begin{tabular}{|c|c|c|c|c|c|}
\hline & $\begin{array}{l}\text { NN } D E \\
\text { CASOS }\end{array}$ & $\begin{array}{l}\text { EDAD GEST. } \\
\text { [semones] }\end{array}$ & $\begin{array}{c}\text { PESO NAC. } \\
\text { (Mgs) }\end{array}$ & $\begin{array}{c}\text { TAL_A NAC } \\
\text { (cMS) }\end{array}$ & $\begin{array}{l}\text { COCERE SERKCO } \\
(1, \mu \mathrm{g} / \mathrm{d} \mid)\end{array}$ \\
\hline RNT AEG & CB & $39.7 \div 1.2^{\prime \prime}$ & $3.16: 0.32^{\star}$ & $49.1 \div 1.4^{\circ}$ & $537: 5.9^{k}$ \\
\hline R NT PEG & 3 & $39.0 \pm 1.0^{*}$ & $2.18 \div 0.20^{\circ}$ & $46.2 \pm 0.8^{\circ}$ & $520 \pm 6.1^{*}$ \\
\hline RNTGEG & 20 & $40.0 \div 1.0^{x}$ & $3.98 \div 3.2^{\circ}$ & $51.5 \pm 1.1$ & $53.3 \pm 5.4^{*}$ \\
\hline
\end{tabular}

- Dileremcias sogniticativas (p-0.01)

* Sin diferencias signiticalivas

Las caracteristicas de los recién nacidos de pretérmino según el estandar usado ${ }^{15}$ se muestran en la Tabla 4. No se encontraron diferencias significativas en las cupremias, ni en las otras variables.

\section{Tabla 4.}

lidad gestacional, peso, talla y cobre sérico en 27 R.N.preT. según clasificación peso $v_{5}$ edad gestacional. Valores expresados en $\bar{X} \pm$ D.S.

\begin{tabular}{|c|c|c|c|c|c|}
\hline & $\begin{array}{l}\text { NE DE } \\
\text { CASOS }\end{array}$ & $\begin{array}{l}\text { EDAC GEST } \\
\text { (semanos! }\end{array}$ & $\begin{array}{l}\text { PESO NAC } \\
\text { (Mgs) }\end{array}$ & $\begin{array}{c}\text { IALLA NAC } \\
(\subset \mathrm{T} 5)\end{array}$ & $\begin{array}{c}\text { COQRE SERICO } \\
(\mu \mathrm{g} / \mathrm{dl})\end{array}$ \\
\hline RNpreT AES & 24 & $35.1 \pm 2.2$ & $229 \div 0<6$ & $44.4: 2.9$ & $40.1=4.7$ \\
\hline PNpreTPEG & 1 & 36.0 & $1.8 \mathrm{~B}$ & 43.0 & 410 \\
\hline RNDIe'GEG & 2 & $365 \pm 0.7$ & $3.55 \div 0.29$ & $50.3 \div 2.6$ & $45.0 \pm 5.7$ \\
\hline
\end{tabular}

No riubo diterancias signilicolivas

Las concentraciones séricas de cobre de las madres no mostraron correlación significativa con ninguna de las variables matemas consideradas. Tampoco hubo correlación entre la cupremia ma- terna y la de los recién nacidos $(r=-0.087)$.

Se encontró correlación directa $(r=0.707)$ y significativa $(\mathrm{p}<0.00001)$ entre las cupremias de los recién nacidos y sus edades gestacionales (Figura 1). La ecuación de la curva de regresión correspondiente es $Y=-37.71+2.28 X$, donde $Y=$ cobre sérico en reciên nacidos $(\mu \mathrm{g} / \mathrm{dl}$.) y $\mathrm{X}=$ edad gestacional (semanas).

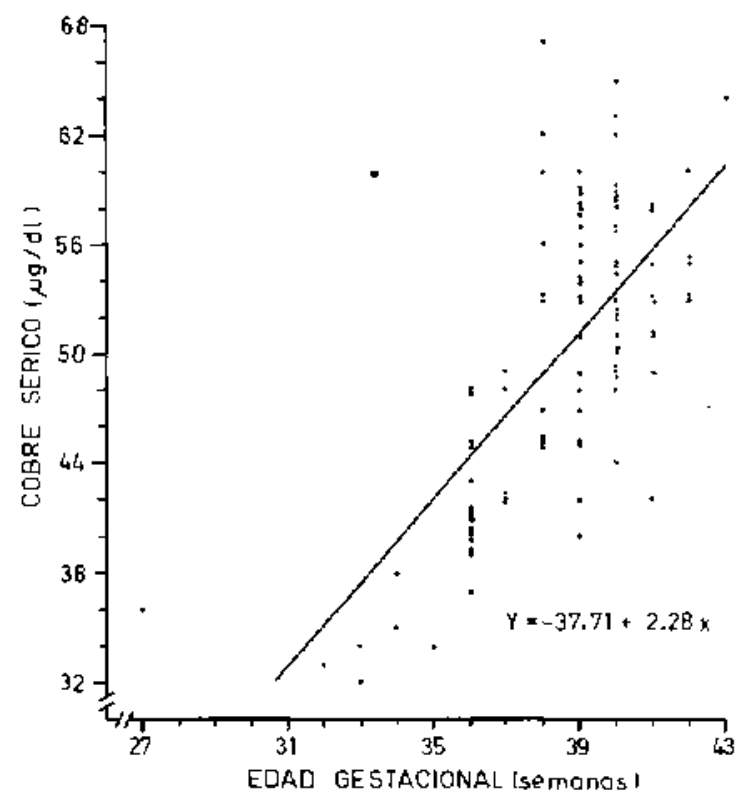

Figura 1.

Línea de regresión del cobre sérico sobre la edad gestacional de los recién nacidos.

La cupremia de los recién nacidos también se correlacionó directa y significativamente con el peso de nacimiento $(\mathrm{r}=0.562)$ y con la talla de nacimiento $(\mathrm{r}=0.586)$. Otras correlaciones significativas ocurriefon entre el peso y la talla de nacimiento $(r=0.911)$; entre el peso y la eded gestacional $(r=0.726)$ y entre la talla y la edad gestacional $(\mathrm{r}=0.791)$.

En el análisis de la información obtenida se realizó una prueba de regresión múltip̀le entre la cupremia y otras variables de los recién nacidos, encontrándose una ecuación estimada que corresponde a:

$\mathrm{Y}=-28.5-0.15 \times$ Talla $+1.66 \times$ Pesu $+2.1 \mathrm{x}$ Edad gestacional; donde

$Y=$ cupremia del tecién nacido $(\mu \mathrm{g} / \mathrm{d} \mathrm{l}$ ) $)$.

No hubo correlaciones significativas entre las concentraciones de cobre sérico en los recién nacidos $y$ otras variables maternas. 


\section{DISCUSION}

En el suero (o plasma) el cobre se encuentra unido a la ceruloplasmina en su mayor parte (93\%), el resto a albúmina $y$, en menor proporcion, a algunos aminoácidos ${ }^{19 \cdot 20}$. Se sabe que el cobre pasa al embrión desde las primeras etapas de la gestación pero se acumula en el hígado fetal principalmente en las últimas 8 a 10 semanas de vida intrauterina ${ }^{3-4}$. Los recién nacidos tienen proporcionalmente mayores concentraciones tisulares de cobre que los adultos, y en un RNT el cobre corporal total alcanza aproximadamente a 16 mg., del que 50 a $75 \%$ se encuentra en el hígado, donde existe en concentraciones 10 a 20 veces superiores a un adulto. Después de nacer, disminuyen las concentraciones hepáticas de cobre al ser usado para el crecimiento ${ }^{1-3}$.

En recién nacidos se han comunicado concentraciones de cobre circulante desde $29 \pm 11 \mu \mathrm{g} / \mathrm{dl}$. hasta $50 \mu \mathrm{g} / \mathrm{dl}$. para RNT $\mathrm{RN}^{13-21 \cdot 22-23}$. En nuestro estudio los RNT presentaron cupremias de $53.7 \pm$ $5.8 \mu \mathrm{g} / \mathrm{dl}$., valores comparables a los de la literatura. El hecho de que los recién nacidos presenten cupremias más bajas que adultos ${ }^{3-7}$ puede ser explicado por la menor concentración de ceruloplasmina circulante propia de los recién naci$\operatorname{dos}^{7 \cdot 20-24}$. Esta proteína incorpora la mayor parte del cobre circulante $y$ al parecer no atraviesa la placenta, siendo principalmente cobre libre el que pasa de la madre al feto por difusión pasiva. Una vez iniciada la vida extrauterina los recién nacidos estarían en condiciones de sintetizar ceruloplasmina y así aumentar sus niveles de cobre circulante $21-22$.

En recién nacidos de pretérmino se ha informado cifras de cobre sérico de $28 \pm 2 \mu \mathrm{g} / \mathrm{dl}$. entre las 28 a 32 semanas de edad gestacional, de $25.1 \pm$ $18.3 \mu \mathrm{g} / \mathrm{dl}$. entre las 31 y 32 semanas y de $33.5 \pm$ $20 \mu \mathrm{g} / \mathrm{dl}$. para 33 a 34 semanas $^{13-25}$. Nuestras cupremias para RNpreT son similares a las descritas.

Las menores concentraciones de cobre circulante en los RNpreT se explicarían porque no tuvieron el tiempo de vida intrauterina necesario para lograr adecuadas concentraciones hepáticas de cobre. Aunque es posible aislar una apoceruloplasmina en el suero de los recién nacidos, no existen evidencias de que los fetos puedan sintetizarla antes del nacimiento ${ }^{19}$. Concomitantemente, los prematuros presentan inmadurez de los mecanismos de síntesis hepática de ceruloplasmina ${ }^{1-20}$ Así los recién nacidos prematuros tienen menores concentraciones de cobre sérico, los que pueden permanecer bajos hasta las 6 a 12 semanas de vidal-25.

En general, las cupremias de los recién naci- dos de este trabajo son semejantes a las de otros estudios. Las variaciones existentes pueden deberse a diferencias en el número de casos estudiados y al empleo de diferentes métodos de análisis y trabajo. Nosotros tomamos muestras por punción venosa, mientras otros autores to hicieron desde el cordón umbilical, donde las cupremias difieren según provengan de la vena o de la arteria $^{20.23}$. Algunos trabajos no tenian como objetivo principal la cuantifjcación de cupremias en el recién nacido y finalmente, en el metabolismo matemo-fetal del cobre se han sugerido diferencias raciales, por factores dietarios y por estado nutricional $\mathbf{1 9 - 2 6}$

No se ha definido en qué medida las cupremias representan el contenido total de cobre corporal. Se ha sugerido que para una mejor cuantificación del cobre total, deberían estudiarse los depósitos tisulares. Se han efectuado mediciones de cobre en pelo, eritrocitos, uñas y cuantificación de ceruloplasmina $^{2 \cdot 19-27}$. No obstante, la determinación de cobre en suero o plasma sigue siendo la más utilizada.

Según nuestros resultados, la cupremia de los recién nacidos estaria influenciada y en gran medida determinada por la edad gestacional. Los prematuros presentaron menores cifras de cobre sérico que los RNT, corroborando la información existente. En la Tabla 2, la ausencia de diferencias significativas para el cobre sérico entre las categorías 27 a 33 y de 34 a 37 semanas, puede deberse al tipo de prueba estadistica y al bajo número de casos en la primera categoría. Igual explicación es atribuible a la falta de diferencias significativas observadas en los datos de la Tabla 4.

La edad gestacional de los recién nacidos mostró una correlación significativa con el cobre sérico, aunque también el peso y la talla se correlacionan con las cupremias. Resulta difícil separar esas tres variables en su relación con la cupremia de los recién nacidos, ya que además están correlacionadas entre ellas. Sin embargo, la edad gestacional modifica sustancialmente la concentración de cobre sérico y presenta la más alta correlación con la cuprenia de los recién nacidos. A su vez, en una determinada edad gestacional, el peso de nacimiento no induce diferencias en la cupremia como se puede desprender de los resultados de la Tabla 3.

Las concentraciones séricas de cobre encontradas en las madres $(234.3 \pm 39.1 \mu \mathrm{g} / \mathrm{dr}$.) resultaron ser bastante mayores que las comunicadas para adultos normales. Shaw ${ }^{3}$ describió cupremias de $107 \pm 23 \mu \mathrm{g} / \mathrm{dl}$. para mujeres adultas normales, mientras que Ashkenazi y col. ${ }^{7}$ obtuvieron valores entre 120 y $160 \mu \mathrm{g} / \mathrm{dl}$. para adultos en general.

Otros autores han informado concentraciones 
que oscilan, entre los $85 y \operatorname{los} 163 \mu \mathrm{g} / \mathrm{dl} .^{10-28}$. Sin embargo, se han descrito cupremias maternas que van desde $139.5 \pm 25.4 \mu \mathrm{g} / \mathrm{dl}$. para madres de RNpreT y $150 \pm 25$ para madres de RNT $^{13}$, hasta $177 \pm 0.36 \mu \mathrm{g} / \mathrm{dl}$. para madres en los 3 primeros dias post parto 21 , mientras que Chez y col. afirman que la cupremia materna puede llegar a ser $\tan$ alta como $350 \mu \mathrm{g} / \mathrm{dl}^{29}$. Nuestros hallazgos se aproximan más a los descritos por Henkin y col. ${ }^{22}$ para 15 madres con embarazos de término y que corresponden a $221 \pm 53 \mu \mathrm{g} / \mathrm{dl}$.

El cobre circulante aumenta en el embarazo a partir del 2 do. trimestre, debido principalmente a mayor sintesis de ceruloplasmina ${ }^{21-23-30}$. Este aumento en ceruloplasmina sería secundario a los ajustes hormonales, como aumento de estrógenos, que producen durante el curso del embarazo. No obstante, Kundu y col. ${ }^{23}$ en un estudio prospectivo no encontraron correlación entre la cupremia materna y los niveles de estriol y estetrol endógenos.

Podemos concluir de nuestros resultados que los recién nacidos presentan bajas concentraciones de cobre sérico, siendo estas aún menores en los prematuros. Las cupremias se correlacionan con la edad gestacional y con el peso y la talla de nacimiento, sin embargo, la edad gestacional sería la principal determinante. Los prematuros por sus menores reservas de cobre, estarian susceptibles a presentar hipocupremia durante su desarrollo. Establecer su significado clínico y fisjopatológico requiere de mayores estudios.

\section{RESUMEN}

Se determinaron valores de cobre sérico en 99 recién nacidos y sus respectivas madres. Analizados los resultados según edad gestacional, los recién nacidos de 38 o más semanas, presentaron cupremias de $53.7 \pm 5.8 \mu \mathrm{g} / \mathrm{dl}$. ( $\mathrm{x} \pm$ D.S.); los de 34 a 37 semanas, $41.7 \pm 4.1 \mu \mathrm{g} / \mathrm{dl}$. y los de 27 a 33 semanas, $33.8 \pm 1.7 \mathrm{\mu g} / \mathrm{dl}$. El valor obtenido en los RNT difiere significativamente $(p<0.01)$ del obterido en los prematuros. Hubo correlación directa $(r=0.707)$ entre la cupremia y la edad gestacional de los recién nacidos, influyendo además la talla y el peso. No se presentaron diferencias significativas en las cupremias según sexo ni tampoco entre recièn nacidos de diferentes pesos para una misma edad gestacional. Tampoco existió correlación entre las cupremias maternas y del recién racido. Los RNpreT tendrían niveles de cobre sérico más bajos y estarían expuestos a presentar hipocupremias en su desarrollo.

\section{REFERENCIAS}

1 Walravens, P.A.- Nutritional importance of copper and zinc in neonates and infants. Clin. Chem. 26(2): 185, 1980.

2 Solomons, N.W.: On the assessment of zinc and copper nutriture in man. Am. J. Clin. Nutr. 32(4): B56, 1979.

${ }^{3}$ Shaw, J.C.Ln: Trace elements in the fetus and young infant. Am. J. Dis. Chil. L34(1): 74, 1980.

4 Castillo, C.; Fisberg, M.; Laty, R.: El cobre como mineral esencial para la nutrición humana. Rev. Chil. Ped. 53(3): 268, 1982.

5 Sandstead, H.H.: Copper bioavailability and requirements. Ann. J. Clin. Nutr. 35(4): 809, 1982.

${ }^{6}$ Al - Rashid, RA.; Spangler, J.: Neonatal copper deficiency. New Eng. J. Med-285(15): 841, 1971.

${ }^{7}$ Ashkenozi, A.; Levin, $S_{\rightarrow}$ Djaldetti, M.; Fishet, $E_{\text {; }}$ Benvenssi, $D$.: The syndrome of neonatal copper deficiency. Pediatrics 52(4): 525, 1973.

8 Singsubramanian, KN.; Henkin. R.l.: Behavioral and dematologic changes and low serum zinc and copper concentrations in two premature infants after parenteral alimentation. J. Pediatr. 93(5): 847, 1978.

${ }^{9}$ Heller, R.M.; Kirchner, S.G.: ONeill, JA.; Hough, A.J.; Howard, L.; Kramer. S.S.; Green, HLL.: Skeletal changes of copper deficiency in infants receiving prolonged total parenteral nutrition. J. Pediatr. 92(6): $947,1978$.

10 Navel, $Y_{\text {; }}$ Hazani, A.; Berant. M.: Copper deficiency with cow's milk diet. Pediatrics 68(3): 397, 1981 .

11 Fisberg, M.; Castitlo, C.; Egaña, J.I.; Uauy, $R$.. Factores condicionantes de hipocupremia en lactantes: marásmicos. Rev. Chil. Ped. 52(55): 410, 1981.

12 Puentes, $R_{\text {.; }}$ Uauy, $R$; Castillo, $C$.: Déficit de cobrc en el lactante. Rev. Chit. Ped. 53(2): 144, 1982.

13 Hilman, L.S.: Scrial serum copper concentrations in premature and SGA infants during the first 3 month of life. J. Pediatr. 98(2): 305, 1981 .

14 Hillmon, L.S.i Martin, L.: Fiore, B.: Eftect of oral copper supplementation on serum copper and ceruloplasmina concentrations in premature infants. J. Pediatr. 98(2): $31 \mathrm{~J}, 198 \mathrm{~L}$.

15 Battaglia, F.: Lubchenco L.D.: A practical classification of newbron infants by weight and gestational age. J. Pcdiatr. 71: 159, 1967.

16 Sprague, J,; Slavin C.: Determination of iron copper and zinc in blood sertm by an atomic absorption method requiring only dilution. Atomic Absorption Newslet ter 4: 228, 1965.

17 Domenech $i$ Massons, J.: Bioestadística. Métodos estad isticos para investigadores. Editorial Herder, Barcelona, 1977.

18 Jeliffe, D.B.: Evaluación del estado nutricional de la comunidad. Organización Mundial de la Salud. Ginebra, 1968.

19 Hatano. $S_{\text {; }}$ Nishi, $Y_{;}$Uszit, $T_{r}$. Copper levels in plasma and erythrocytes in healthy Japanex children and adults. Ann. J. Clin. Nutr. 35(I): 120, 1982.

20 Mason, KE.: A conspectus of research on copper metabilism and requirements of man. J. of Nutrition 109(11): 1979, 1979.

21 Vi. S. $C_{. ;}$Love, A.H.G.; Thompson, $W_{.:}$Serum and hair concentuations of copper during pregnancy. Am. J. Clin. Nutr. 34: 2382, 1981

22 Henking, R.l.: Marshal, J.R.; Meret, S.: Maternal fetal metabolism of copper and zinc at term. Am. I. Obstef. Gynecol. 1 10(1): 131, 1971.

${ }^{2} 3$ Kundu, N.; Wachs, M.; fverson, G.B.; Petersen, L.P.: Fetal and maternal serum copper levels before and 
during labor in normal and complicated pregnancies. Obstet. Gynecol. 57(3): 313, 1981.

24 Henking, R.I.; Schulman, J.D.i Schulmon. C.B.; Bronzet, D.A.: Changes in total, nondiffusible, and diffusible plasna zinc and copper during infancy. J. Pediatr. 82(5): 831, 1973.

25 Manser, J.J.; Crawford, C.S.; Tyrala, E.E.: Brodsky, N.L.; Grover, W.D.: Serum copper concentrations in sick and well preterm infants. J. Pediatr. 97(5): 795, 1980.

${ }^{26}$ Holt. A.B.; Spargo, R.M.; Iverson, J.B. Faulkner, GSS: Cheek, D.B.: Serum and plasma zinc, copper and iron concentrations in Aboriginal communities of North Western Australia. Am. J. Clin. Nutr. 33(1): 119, 1980.

27 Gibson, R.S.: De Wolfe, M.S.: Copper, zinc, manga- neso, vanadium, and iodine concentrations, in the hair of Canadian low birth weight teonates. Am. J. Clin. Nutr. 32: 1728, 1979.

28 Shike, M.; Roulet, M.; Kurian, $R_{r}$. Whitwell, $J_{\text {.; }}$ Stewart, $S_{\text {.; }}$ Jeejeebhoy, $K$ N.: Copper metabolism and requirements in total parenteral nutrition. Gastroenterology $81(2): 290,1981$.

${ }^{29}$ Chez, R.A.; Henking, RJ.; Fox, R.: Amniotic fluid copper and zinc concentrations in human pregnancy. Obstet. Gynecol. 52(1): 125, 1978.

${ }^{30}$ Fattah, M.M.A.; Brahim, F.X.; Ramadan, M.A.: Sammour, $M . B .:$ ceruloplasmin and copper levels in maternal and cord blood and in the placenta in normal pregnancy and in pre-eclampsia. Acta Obstet. Gynecot. Scand. 55(5): 383, 1976. 